\title{
Styling without Shedding: Novel Topical Formula Reduces Hair Shedding by Contracting the Arrector Pili Muscle
}

John McCoy*, Andy Goren, Maja Kovacevic, Mirna Situm, Andrija Stanimirovic, Jerry Shapiro, and Rodney Sinclair

*Author whom correspondence should be addressed (johnm@appliedbiology.com)

\section{Author Affiliations:}

John McCoy, PhD: Applied Biology, Irvine, California, USA.

Andy Goren, MD: Applied Biology, Irvine, California, USA. Department of Dermatology and Venereology, University of Rome "G.Marconi", Italy.

Maja Kovacevic, MD: Department of Dermatology and Venereology, University of Rome "G.Marconi", Rome, Italy.

Mirna Situm, MD: Department of Dermatology and Venereology, University Hospital Center "Sestre milosrdnice", Zagreb, Croatia.

Andrija Stanimirovic, MD, PhD: Department of Clinical Medicine, University of Applied Health Sciences, Zagreb, Croatia

Jerry Shapiro, MD: Department of Dermatology, New York University Langone Medical Center, New York City, New York, USA.

Rodney Sinclair, MD: Department of Medicine, University of Melbourne, Victoria, Australia.

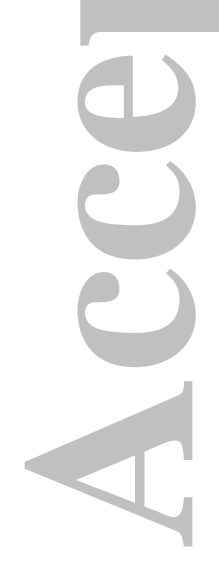

This is the author manuscript accepted for publication and has undergone full peer review but has not been through the copyediting, typesetting, pagination and proofreading process, which may lead to differences between this version and the Version record. Please cite this article as doi:10.1111/dth.12575. 


\section{ABSTRACT}

Approximately $40 \%$ of women experience excessive hair shedding during styling (e.g., hair brushing). Previously, we demonstrated that topically applied phenylephrine, a potent $\alpha_{1}$ adrenergic receptor agonist, can be used to contract the arrector pili muscle of the follicular unit; thus, increasing the force required to pluck hair and reducing shedding during brushing. While demonstrating efficacy, phenylephrine has several drawbacks when applied to the scalp, including the possibility cardiovascular events. We hypothesized that a high concentration of a weak $\alpha_{1}$ agonist would allow for: (1) rapid penetration through the stratum corneum eliciting a quick response; (2) a low probability of cardiac adverse events owing to the low receptor binding affinity; and (3) an efficacy of the weak $\alpha_{1}$ agonist similar to that of phenylephrine at the local site of application. Accordingly, we developed a novel topical solution, AB-102, containing a high concertation of a weak $\alpha_{1}$ agonist. Several studies were conducted to test the safety and efficacy of AB-102. In a dose escalating safety study, utilizing a wearable holter monitor, we observed no cardiac or hemodynamic adverse events. In addition, in a controlled efficacy study, AB-102 reduced the number of hairs shed during brushing by up to $77 \%$ (average of $38 \%$ ).

\section{INTRODUCTION}

Human hair is an important aspect of a woman's self image. Therefore, it is not surprising that changes in hair density are often discussed in dermatological consults. Hair shedding is a normal part of the physiological processes of the body. Normally, women shed between 50 to 150 hairs in a 24-h period, while shedding in excess of 150 hairs can indicate thinning (1). To aid physicians in their assessment of shedding, a visual-analog scale was developed, the Sinclair Hair Shedding Scale (2). In a survey of 300 subjects visiting a public hospital for conditions other than alopecia, approximately $40 \%$ reported experiencing excessive hair shedding as defined by the Sinclair Hair Shedding Scale (3).

Increased hair shedding occurs as a result of various conditions such as stress, endocrinological and nutritional disorders, inflammatory and infectious disorders, and after the administration of certain drugs (4). It is also present in different dermatological disorders including anagen effluvium, telogen effluvium, cicatricial alopecia, female pattern hair loss, alopecia areata, and traction alopecia (4). Traction alopecia results from persistent pulling of the roots of hair over time leading to bald spots or diffuse telogen shedding (5). While traction alopecia would be considered an extreme case, increased shedding in women can also result from many of the mechanical hair procedures commonly used during hair styling. For example, blow drying, flat ironing, hair curling, and brushing all apply traumatic force to hair follicles and consequently remove hair.

Each hair follicle in the scalp contains an arrector pili muscle, a smooth muscle attached at one end to the hair follicle and at the other to the inner surface of the basal layer of the epidermis. Its contraction produces piloerection, which can be stimulated directly by mechanical, thermal, electrical, or pharmacological stimuli or indirectly via an axon reflex (6). Piloerection occurs when catecholamines bind to $\alpha_{1}$ adrenergic receptor ( $\alpha_{1^{-}}$ AR), a G-protein coupled receptor, expressed on the arrector pili muscle. Because the 
force applied to the hair shaft, during contraction of the arrector pili, is parallel and in the opposite direction of epilation, stimulation of piloerection in the scalp greatly increases the threshold of force required to pluck hair.

In a pilot study of 15 female subjects, we demonstrated that topically applying the $\alpha_{1}$ adrenergic receptor agonist, phenylephrine hydrochloride, increased the threshold of force required to pluck hair by $172 \%$. Additionally, topical phenylephrine reduced shedding during brushing by $40 \%$ (7). During the study, subjects applied $0.5 \mathrm{~mL}$ of a $10 \%$ phenylephrine solution to an $8 \times 10 \mathrm{~cm}$ target area. The size of the target area was chosen to insure that the total dosage stayed below our calculated safety margin. Phenylephrine, a classic selective $\alpha_{1}$-AR agonist, was chosen as the investigational drug because of its long and established safety profile. While demonstrating efficacy, $\checkmark$ phenylephrine has several potential drawbacks if applied to the entire scalp, including accidental ocular exposure resulting in prolonged pupil dilation and the possibility of dose dependent cardiovascular adverse events.

To circumvent the limitations of phenylephrine we developed a novel formulation using synephrine. Synephrine has been studies extensively in its oral form and has not been shown to induce significant cardiac or hemodynamic changes (8). Synephrine is an isomer of phenylephrine, different only in the position of the phenolic hydroxyl group; phenylephrine has a meta-hydroxyl, while synephrine has a para-hydroxyl. Synephrine is a weak $\alpha_{1}$-AR agonist, exhibiting a potency approximately 150 fold less that of phenylephrine (9). As such, a higher concentration was needed to observe the same $\alpha_{1}$ AR activation on the scalp. We hypothesized that this would be advantageous for two reasons: First, the higher concentration of the active would facilitate penetration through the stratum corneum (10). Second, owing to the low $\alpha_{1}$-AR binding affinity (11, 12), synephrine would not likely cause systemic effects once diluted outside of the local application site. The novel formula, AB-102, was tested in a randomized controlled study similar to our previous work with phenylephrine (7); cardiac and hemodynamic changes were also tracked to assess safety.

\section{METHODS}

Patients: 40 female subjects, ages 18-40, were included in the study. Subjects were Caucasian women having medium to long hair. Subjects were recruited based on their frequent use of strenuous mechanical hair procedures during hair styling. Subjects with uncontrolled hypertension, that were pregnant or breastfeeding, had been diagnosed with pattern hair loss or with other hair loss in conjunction with female pattern hair loss were excluded from the study. The study was conducted with the approval of the hospital ethics committee. All subjects gave informed consent during enrolment in the study.

Hair Removed During Styling: To measure hair loss during cosmetic procedures an intrasubject design was used. Hair was parted in the middle of the scalp to divide the left and right sides. To the right side of the scalp, $2 \mathrm{~mL}$ of the investigational solution (AB-102) was applied by the principal investigator and massaged until dry. After the applied solution was dry, another $2 \mathrm{~mL}$ of the investigational solution was applied by the principal investigator and massaged until dry. The left side of the scalp was left 
untreated. 10 minutes after the second application of the investigational solution, the subjects washed and dried their hair. Hair was dried with an electric hair dryer.

After washing and drying their hair, subjects were given two new brushes (Skelettbürste, 1 St, Ebelin, Germany) sealed in individual plastic bags. Subjects were placed on a large white paper mat. They were instructed to brush the right side of the scalp 20 times with the brush removed from the bag labeled "right". They were instructed to brush from the front of the scalp to the base of the neck in one continuous stroke. After brushing 20 times, the brush was returned to the bag and sealed. Any hair that was visible on the white mat was added to the bag. The same procedure was conducted on the left side of the scalp with the brush sealed in a bag labeled "left". Hairs from each brush and bag were removed and counted manually at a later time.

Epilation Force: To evaluate the effect of AB-102 on the force required to pluck hairs from the scalp, a hand-held spring dynamometer, or "trichotillometer" was used (13). The trichotillometer records the maximum force threshold, in grams, required to pluck a single hair from the scalp; the performance and statistical variance of the instrument have been previously reported (13-15). We performed force measurements using the trichotillometer on 20 subjects.

At the beginning of the study 10 hairs were plucked using the trichotillometer from the frontal region of the scalp and the force required for each pluck was recorded. Afterward, $5 \mathrm{~mL}$ of the investigation solution was applied by the principal investigator to the entire scalp and massaged thoroughly, until it was dry. A second application of 5 $\mathrm{mL}$ of the investigational solution was applied and massaged thoroughly. At 30,60, and 120 minutes following the application of the solution, 10 hairs were plucked using the trichotillometer from the frontal region of the scalp and the force required for each pluck was recorded.

Cardiac and Hemodynamic Assessment: To evaluate the effect of AB-102 on systemic hemodynamic parameters, a holter monitor (Contec, TLC500) was placed on each subject one hour prior to participating in the epilation force study. At baseline, blood pressure and heart rate were recorded. During the epilation force study, described above, each subject applied a total of $10 \mathrm{~mL}$ of AB-102 to the entire scalp and massaged thoroughly. After completing the epilation force study, subjects were instructed to refrain from washing their hair for a minimum of 8 hours. Blood pressure, heart rate, and echocardiograms (ECG) data were collected over a 24-hour period via the holter monitor. After 24 hours, the subjects returned to the clinic and the holter monitors were collected. Blood pressure and heart rate data were collected by the principal investigator. ST segment changes and abnormal arrhythmias as determined by the ECG were assessed by an independent cardiac expert. In total 20 subjects were studied.

\section{RESULTS}

After tabulating the data of 40 subjects studied in the hair shedding experiment (Table 1), we found a decrease in hair loss in 30 out of 40 subjects $(75 \%)$ in the target area (right half of the scalp) following the application of AB-102 compared to the untreated side. The range of individual reductions had a large variance (-180\% to $77 \%)$, however, the average reduction in hair loss was $38 \%$ for the cohort of 40 subjects. Measurements 
of the epilation force threshold in 20 subjects showed similar improvements (Table 2). The epilation force threshold on scalp hair follicles increased $20 \%, 20 \%$, and $22 \%$ on average (range: $-26 \%$ to $82 \%$ ) following the application of AB-102 at 30, 60, and 120 minutes, respectively. Hemodynamic parameters recorded over a 24-hour period demonstrated no significant changes, suggesting the new formula does not have systemic effects. Independent assessment of each subject's ECG confirmed that there were no ST segment changes or abnormal arrhythmias during the 24 hours sampled.

\section{DISCUSSION}

In our previous work, we demonstrated that the $\alpha_{1}$-AR agonist phenylephrine increased the epilatory force required to pluck hair and consequently reduced shedding during hairstyling. In this communication, we have demonstrated a novel formula, AB-102, has similar efficacy. AB-102 reduced the number of hairs lost during brushing by $38 \%$ in our cohort of 40 women with a maximum reduction of $77 \%$. Additionally, the threshold force for epiliation was increased by at least $20 \%$ over the two hours measured after the application of $\mathrm{AB}-102$. The maximum increase in force measured was $86 \%$. No force measurements were made beyond two hours after the application of AB-102. In future studies, we intend to elucidate the maximum time AB-102 can provide protection.

Blood pressure, heart rate, and ECG were collected during the course of the study. Data was measured continuously for 24 hours post application of AB-102 using a holter monitor. No cardiac or hemodynamic changes were observed in the 20 subjects sampled. This data supports our hypothesis that due to the low $\alpha_{1}$-AR binding affinity of synephrine, activation of $\alpha_{1}$-AR is not likely outside of the local application site (where the concentration of synephrine is very high).

Many women experience excessive shedding during routine hairstyling. The force from common practices, e.g., brushing, flat ironing, and washing, removes hair from the root. In this study we have demonstrated AB-102 can reduce this phenomena safely and effectively.

\section{REFERENCES}

1. Sinclair R. Diffuse hair loss. Int J Dermatol. 1999; 38: 8-18.

2. Sinclair R. Hair shedding in women: how much is too much? Br J Dermatol. 2015; 173(3): 846-848.

3. Kovacevic M, Goren A, Shapiro J, Sinclair R, Lonky NM, Situm M, Bulat V, Bolanca Z, McCoy J. Prevalence of hair shedding among women. Dermatol Ther. 2017; 30(1): Epub 2016 Oct 6.

4. Malkud S. Telogen Effluvium: A Review. J Clin Diagn Res. 2015; 9: WE01-3.

5. Ozçelik D. Extensive traction alopecia attributable to ponytail hairstyle and its treatment with hair transplantation. Aesthetic Plast Surg 2005; 29(4): 325-327.

6. Siepmann T, Gibbons CH, Illigens BM, Lafo JA, Brown CM, Freeman R. Quantitative pilomotor axon reflex test: a novel test of pilomotor function. Arch Neurol. 2012; 69(11): 1488-1492.

7. Goren A, Shapiro J, Sinclair R, Kovacevic M, McCoy J. $\alpha 1$-AR agonist induced piloerection protects against the development of traction alopecia. Dermatol Ther. 2016; 29: 160-163. 
8. Stohs SJ, Preuss HG, Shara M. A review of the receptor-binding properties of psynephrine as related to its pharmacological effects. Oxid Med Cell Longev. 2011; 482973. doi: 10.1155/2011/482973. Epub 2011 Aug 1.

9. C. M. Brown, J. C. McGrath, J. M. Midgley, A. G. Muir, J. W. O’Brien et al., "Activities of octapamine and synephrine stereoisomers on alpha-adrenoreceptors," British Journal of Pharmacology 1988; 93: 417-429.

10. Benson HA. Transdermal drug delivery: penetration enhancement techniques. Curr Drug Deliv. 2005; 2(1): 23-33.

11. G. Ma, S. A. Bavadekar, B. T. Schaneberg, I. A. Khan, and D. R. Feller, "Effects of synephrine and $\beta$-phenethylamine on human $\alpha$-adrenoceptor subtypes," Planta Medica. 2010; 76(10): 981-986.T. Hibino, M. Yuzurihara, Y. Kase, and A. Takeda, "Synephrine, a component of Evodiae fructus, constricts isolated aorta via adrenergic and serotonergic receptors," Journal of Pharmacological Sciences 2009; 111(1): 73-81.

12. Wyness LA, McNeill G, Prescott GL. Trichotillometry: the reliability and practicality of hair pluckability as a method of nutritional assessment. Nutr J 2007; 6: 9.

13. Chase ES, Weinsier RL, Laven GT, Krumdieck CL. Trichotillometry: the quantitation of hair pluckability as a method of nutritional assessment. Am J Clin Nutr 1981; 34(10): 2280-2286.

14. Smelser DN, Smelser NB, Krumdieck CL, Schreeder MT, Laven GT. Field use of hair epilation force in nutrition status assessment. Am J Clin Nutr 1982; 35: 342346.

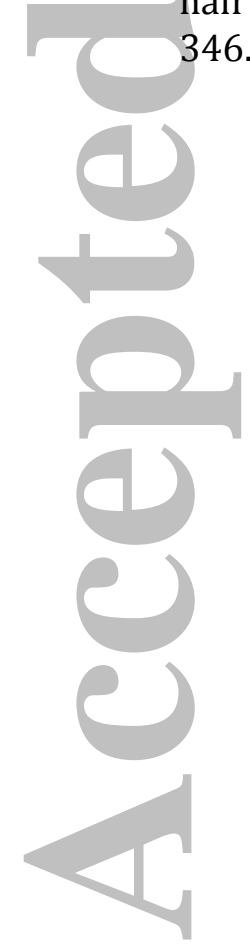




\begin{tabular}{|c|c|c|c|}
\hline \multirow{2}{*}{ Subject \# } & \multicolumn{3}{|c|}{ Number of Hairs Removed with Brush } \\
\hline & Control & AB-102 & Reduction \\
\hline 1 & 18 & 7 & $61 \%$ \\
\hline 2 & 43 & 33 & $23 \%$ \\
\hline 3 & 21 & 5 & $76 \%$ \\
\hline 4 & 46 & 19 & $59 \%$ \\
\hline 5 & 39 & 39 & $\mathbf{0 \%}$ \\
\hline 6 & 34 & 12 & $65 \%$ \\
\hline 7 & 7 & 8 & $-14 \%$ \\
\hline 8 & 47 & 22 & $53 \%$ \\
\hline 9 & 20 & 9 & $55 \%$ \\
\hline 10 & 12 & 9 & $25 \%$ \\
\hline 11 & 2 & 2 & $\mathbf{0 \%}$ \\
\hline 12 & 24 & 18 & $25 \%$ \\
\hline 13 & 7 & 2 & $71 \%$ \\
\hline 14 & 7 & 9 & $-29 \%$ \\
\hline 15 & 9 & 3 & $67 \%$ \\
\hline 16 & 7 & 4 & $43 \%$ \\
\hline 17 & 3 & 1 & $67 \%$ \\
\hline 18 & 15 & 10 & $33 \%$ \\
\hline 19 & 14 & 6 & $57 \%$ \\
\hline 20 & 13 & 9 & $31 \%$ \\
\hline 21 & 35 & 23 & $34 \%$ \\
\hline 22 & 6 & 7 & $-17 \%$ \\
\hline 23 & 46 & 28 & $39 \%$ \\
\hline 24 & 10 & 18 & $-80 \%$ \\
\hline 25 & 52 & 12 & $77 \%$ \\
\hline 26 & 20 & 13 & $35 \%$ \\
\hline 27 & 4 & 6 & $-50 \%$ \\
\hline 28 & 23 & 18 & $22 \%$ \\
\hline 29 & 11 & 7 & $36 \%$ \\
\hline 30 & 5 & 14 & $-180 \%$ \\
\hline 31 & 21 & 17 & $19 \%$ \\
\hline 32 & 6 & 2 & $67 \%$ \\
\hline 33 & 23 & 23 & $0 \%$ \\
\hline 34 & 8 & 6 & $25 \%$ \\
\hline 35 & 10 & 8 & $20 \%$ \\
\hline 36 & 3 & 1 & $67 \%$ \\
\hline 37 & 2 & 2 & $0 \%$ \\
\hline 38 & 4 & 1 & $75 \%$ \\
\hline 39 & 20 & 8 & $60 \%$ \\
\hline \multirow[t]{4}{*}{40} & 33 & 10 & $70 \%$ \\
\hline & \multicolumn{2}{|c|}{ Total } & Reduction \\
\hline & Control & AB-102 & \\
\hline & 730 & 451 & $38 \%$ \\
\hline
\end{tabular}

Table 1. Number of hairs removed with brush after the application of AB-102. 


\begin{tabular}{|c|c|c|c|c|c|c|c|}
\hline \multirow[b]{2}{*}{ Subject \# } & \multicolumn{4}{|c|}{ Grams of Force [Average] } & \multicolumn{3}{|c|}{ Percent Increase } \\
\hline & Baseline & $\begin{array}{c}30 \\
\text { min. }\end{array}$ & $\begin{array}{c}60 \\
\text { min. }\end{array}$ & $\begin{array}{l}120 \\
\text { min. }\end{array}$ & $\begin{array}{c}30 \\
\text { min. }\end{array}$ & $\begin{array}{c}60 \\
\text { min. }\end{array}$ & $120 \mathrm{~min}$. \\
\hline 1 & 15.6 & 18.3 & 20.7 & 21.0 & $17 \%$ & $33 \%$ & $35 \%$ \\
\hline 2 & 11.4 & 14.8 & 20.7 & 20.1 & $30 \%$ & $82 \%$ & $76 \%$ \\
\hline 3 & 23.8 & 26.0 & 25.3 & 21.8 & $9 \%$ & $6 \%$ & $-8 \%$ \\
\hline 4 & 21.3 & 23.8 & 26.2 & 31.3 & $12 \%$ & $23 \%$ & $47 \%$ \\
\hline 5 & 11.4 & 18.0 & 16.5 & 17.7 & $58 \%$ & $45 \%$ & $55 \%$ \\
\hline 6 & 20.9 & 28.3 & 23.7 & 25.2 & $35 \%$ & $13 \%$ & $21 \%$ \\
\hline 7 & 17.5 & 30.1 & 30.5 & 29.1 & $72 \%$ & $74 \%$ & $66 \%$ \\
\hline 8 & 22.0 & 29.8 & 31.2 & 26.7 & $35 \%$ & $42 \%$ & $21 \%$ \\
\hline 9 & 29.8 & 31.6 & 22.1 & 24.4 & $6 \%$ & $-26 \%$ & $-18 \%$ \\
\hline 10 & 17.5 & 26.2 & 20.8 & 21.9 & $50 \%$ & $19 \%$ & $25 \%$ \\
\hline 11 & 14.9 & 12.4 & 18.3 & 15.9 & $-17 \%$ & $23 \%$ & $7 \%$ \\
\hline 12 & 20.4 & 27.9 & 22.2 & 25.0 & $37 \%$ & $9 \%$ & $23 \%$ \\
\hline 13 & 15.3 & 18.5 & 13.8 & 19.1 & $21 \%$ & $-10 \%$ & $25 \%$ \\
\hline 14 & 33.8 & 36.6 & 36.8 & 33.0 & $8 \%$ & $9 \%$ & $-2 \%$ \\
\hline 15 & 27.8 & 35.2 & 39.2 & 36.2 & $27 \%$ & $41 \%$ & $30 \%$ \\
\hline 16 & 25.9 & 25.4 & 27.3 & 29.9 & $-2 \%$ & $5 \%$ & $15 \%$ \\
\hline 17 & 32.0 & 33.1 & 33.1 & 35.8 & $3 \%$ & $3 \%$ & $12 \%$ \\
\hline 18 & 30.6 & 35.0 & 34.5 & 35.8 & $14 \%$ & $13 \%$ & $17 \%$ \\
\hline 19 & 26.2 & 26.1 & 31.0 & 30.1 & $0 \%$ & $18 \%$ & $15 \%$ \\
\hline 20 & 20.1 & 27.0 & 31.6 & 33.1 & $34 \%$ & $57 \%$ & $65 \%$ \\
\hline Average & 21.9 & 26.2 & 26.3 & 26.7 & $20 \%$ & $20 \%$ & $22 \%$ \\
\hline
\end{tabular}

Table 2. Grams of force required to pluck hair 30,60, and 120 minutes after the application of $\mathrm{AB}-102$.

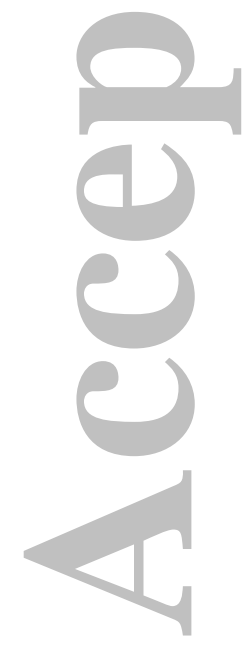




\section{University Library}

\section{- M M N E R VA A gateway to Melbourne's research publications}

Minerva Access is the Institutional Repository of The University of Melbourne

Author/s:

McCoy, J;Goren, A;Kovacevic, M;Situm, M;Stanimirovic, A;Shapiro, J;Sinclair, R

Title:

Styling without shedding: Novel topical formula reduces hair shedding by contracting the arrector pili muscle

Date:

2018-01-01

Citation:

McCoy, J., Goren, A., Kovacevic, M., Situm, M., Stanimirovic, A., Shapiro, J. \& Sinclair, R. (2018). Styling without shedding: Novel topical formula reduces hair shedding by contracting the arrector pili muscle. DERMATOLOGIC THERAPY, 31 (1), https:// doi.org/10.1111/dth. 12575.

Persistent Link:

http://hdl.handle.net/11343/293931 